Annuaire suisse de politique de développement

18 | 1999

La Suisse et l'action humanitaire

\title{
Aide humanitaire, politique de sécurité et prévention des conflits
}

Günther Baechler

\section{OpenEdition}

1 Journals

Édition électronique

URL : https://journals.openedition.org/aspd/668

DOI : 10.4000/aspd.668

ISSN : 1663-9669

Éditeur

Institut de hautes études internationales et du développement

\section{Édition imprimée}

Date de publication : 1 janvier 1999

Pagination : $39-49$

ISSN : 1660-5934

\section{Référence électronique}

Günther Baechler, «Aide humanitaire, politique de sécurité et prévention des conflits », Annuaire suisse de politique de développement [En ligne], 18| 1999, mis en ligne le 12 juillet 2012, consulté le 21 septembre 2021. URL : http://journals.openedition.org/aspd/668; DOI : https://doi.org/10.4000/aspd. 668

(c) The Graduate Institute / Geneva 


\title{
AIDE HUMANITARE, POLITIQUE DE SÉCURITÉ ET PRÉVENTION DES CONFLITS
}

\author{
GÜNTHer BAeCHLER*
}

\section{CONFLITS ET CATASTROPHES HUMANITAIRES À L'AUBE DU XXIE SIÈCLE}

A la fin des années 90, la tendance apparue dès l'issue de la Seconde Guerre mondiale, mais qui s'est nettement affirmée depuis la fin de la guerre froide, se poursuit: les conflits qui éclatent sont de plus en plus souvent intérieurs et touchent avant tout les pays en développement du Sud. Depuis 1989, un tiers environ de tous les pays en développement, y compris les nouveaux Etats indépendants d'Asie centrale, ont été le théâtre de conflits armés. Si l'on ne considère que le groupe des pays les plus démunis, la moitié d'entre eux ont été secoués par un conflit grave. Par ailleurs, il ne faut pas oublier un nombre limité (et qui va décroissant) de conflits armés dans la Caucase, dans les régions d'Europe de l'Est anciennement sous souveraineté soviétique et dans les Balkans. Les guerres, presque toujours intérieures, qui frappent ces régions témoignent de profonds bouleversements dans la société. C'est pourquoi elles se distinguent par les caractéristiques suivantes: longue durée, destruction de l'économie, imprévisibilité politique et grandes souffrances humaines ${ }^{1}$.

Le changement sociopolitique marqué par la violence que traversent actuellement les pays en développement et en transition n'est bien sûr pas sans conséquence pour la politique de développement et pour l'aide humanitaire.

D'une part, la guerre civile est l'adversaire le plus puissant de la coopération au développement. Lorsque la violence se généralise, la coopération au développement risque de se muer en aide à court terme telle que l'aide en cas de catastrophe. Quant à la promotion de la paix, elle est plus souvent utilisée pour stabiliser la situation après une guerre que pour prévenir les crises et la violence. C'est ainsi qu'une part croissante des moyens destinés à la coopération au développement servent à financer la réparation des dégâts causés par la guerre.

Les populations civiles sont les principales victimes des conflits ethnopolitiques, nationalistes, de partage ou motivés par la quête de la puissance. Selon les statistiques du Corps suisse d'aide en cas de catastrophe (ASC), le nombre des conflits armés actuellement en cours a augmenté de 39 à 55 entre 1983 et 1993. Dans le même temps, le nombre des catastrophes naturelles s'est aussi accru pour passer de 50 à 130 et il n'est pas toujours possible de distinguer les séquelles de la guerre de celles d'une catastrophe naturelle ${ }^{2}$. Entre 1980 et 1992, les dépenses que les pays de l'OCDE ont consacrées à l'aide d'urgence bilatérale en raison d'un conflit ou suite à une catastrophe naturelle sont passées de 2 à $6,3 \%$ de l'aide publique au développement de ces pays; en 1994, cette part

* Directeur, Fondation suisse pour la paix, Institut pour la résolution de conflits, Berne.

1. Baechler G., FAST: Frühanalyse von Spannungen und Tatsachenermittlung. Abschlussbericht zum Pilotprojekt FAST, Working Paper 26, Fondation suisse pour la paix, Institut pour la résolution de conflits, Berne, 1997, p. 8.

2. Un seul monde, $\mathrm{n}^{\circ} 2$, juin 1998 , p. 12. 
avait déjà atteint $10 \%$. Depuis lors, la tendance est à une légère baisse. Plus concrètement, cela signifie que les Etats de l'OCDE ont consacré 500 millions de dollars à l'aide humanitaire en 1988 et une somme sept fois plus élevée, soit 3,5 milliards de dollars, à peine cinq ans plus tard (1993) ${ }^{3}$. Comme le regrette le Ministère allemand pour la coopération économique (BMZ) dans un rapport, «on prive ainsi la coopération au développement des moyens destinés à ses propres objectifs, c'est-à-dire à la mise sur pied de structures à long terme pour assurer un développement durable et autonome ${ }^{4}$.

D'autre part, le transfert des moyens de la coopération au développement à long terme, dans le sens d'une prévention structurelle des crises, vers une aide humanitaire d'urgence et vers l'aide à la reconstruction, à relativement court terme, n'est qu'un aspect des choses. Le second aspect comprend des «interventions humanitaires" encore plus coûteuses que sont les opérations dites de maintien de la paix dans le cadre de l'ONU. L'année 1994 a constitué un record dans ce domaine, puisque 78'000 personnes étaient alors engagées dans des actions de maintien de la paix de l'ONU dont le coût s'est élevé à quelque 4 milliards de dollars ${ }^{5}$. Cette année-là, la guerre a causé la mort de plus de 4 millions de personnes et jeté, selon les estimations du HCR, 27 millions d'autres personnes sur les routes.

Les actions humanitaires, aussi bien l'aide que les interventions, ont dû viser en premier lieu les régions où les conflits armés locaux, les guerres civiles, les crises politiques et les génocides entravaient les activités sociales, économiques et politiques courantes. L'aide et les interventions ont donc dû être menées à bien dans un contexte difficile, voire précaire. Dans le courant des années 90 , cette situation a eu des effets différents sur les deux aspects des actions humanitaires.

Dans le présent article, nous expliquons que ce sont justement les tendances opposées du maintien de la paix, d'une part, et de l'aide humanitaire, d'autre part, qui jouent un rôle prépondérant. Dans ce contexte, l'aide humanitaire est plus que jamais soumise à des contraintes qui relèvent de la politique de sécurité. Pour illustrer notre propos, nous commencerons par examiner l'évolution internationale. Nous présenterons ensuite le débat qui a lieu en Suisse, notamment dans le cadre du rapport Brunner. Pour terminer, nous mettrons l'accent sur la prévention des conflits, car seule une politique de prévention parviendra en fin de compte à réduire la pression qui pèse sur l'aide d'urgence.

2. APAISER LES SOUFFRANCES ET GARANTIR LA PAIX: DE LA JUSTIFICATION HUMANITAIRE DE LA POLITIQUE DE SÉCURITÉ À LA MOTIVATION SÉCURITAIRE DE L'AIDE HUMANITAIRE

\section{$\square$ Les interventions humanitaires dans les années 90}

L'envergure et la diversité des interventions humanitaires ont atteint leur maximum avec la présence de la FORPRONU en ex-Yougoslavie en 1994 et 1995.

3. Bundesministerium für Zusammenarbeit (éd.), «Entwicklungszusammenarbeit und Krisenvorbeugung», $B M Z$ aktuell, Bonn, juin 1997, p. 2.

4. Schmieg E., «Entwicklungszusammenarbeit und Krisenvorbeugung», Bonn, 1995 (manuscrit), p. 2.

5. Le coût des 18 missions de l'ONU menées l'année précédente a atteint 3,6 milliards de dollars. Voir à ce propos Lund M.S., Preventing Violent Conflicts: A Strategy for Preventive Diplomacy, Washington D.C., 1996, pp. 22 et suiv. 
Une alliance, parfois risquée, d'interventions civiles et militaires dans les crises internes avait pour but d'apaiser les souffrances des populations civiles et de prévenir une menace pour la paix mondiale, conformément au chapitre VII de la Charte des Nations Unies. De plus, les interventions civiles et militaires ont conféré une importance croissante à la motivation humanitaire des décisions du Conseil de sécurité. Voici quelques exemples d'interventions à forte intensité de coût ou de personnel qui ont été opérées à l'époque:

- surveillance ou organisation d'élections dans des pays ou dans des régions non encore entièrement pacifiés (en Angola et au Cambodge par exemple);

- protection des habitants - minoritaires ou majoritaires - d'une région contre une menace de violence, comme ce fut le cas dans les trois zones protégées par les Nations Unies (ZPNU) en Croatie et lors de l'opération Safe Heaven («Ciel sûr») destinée à protéger les Kurdes dans le nord de l'Irak;

๖ création et contrôle de zones de sécurité, par exemple dans les villes bosniaques, contre les attaques militaires;

- garantie de la démilitarisation (partielle) de certaines zones, par exemple les environs de Sarajevo et de Goradze en Bosnie;

๖ transport et distribution de l'aide humanitaire matérielle et autres mesures humanitaires (santé, prisonniers, services psychosociaux) pendant les phases critiques d'un conflit (de telles opérations ont été menées à grande échelle en Somalie);

- soutien au rétablissement du bon fonctionnement gouvernemental (police) après une guerre civile (Salvador et Cambodge);

- surveillance et rapports sur le respect du droit public international par les parties au conflit, ainsi que sur le respect des droits de l'homme pendant et après un conflit (Rwanda) 6 .

Après cette phase d'expansion, qui a culminé en 1995, ce n'est pas le nombre, mais la taille, le coût et la complexité des missions qui ont rapidement diminué. A la fin du mois de décembre 1997, moins de 15'000 personnes, militaires et policiers civils confondus, étaient encore engagées dans des interventions. De plus, les coûts ne dépassaient pas 1,3 milliard de dollars. A titre de comparaison, l'opération menée au Mozambique après la guerre civile (retour des réfugiés, démobilisation des forces armées, reconstruction des infrastructures et soutien au budget national) a coûté à elle seule quelque 2 milliards de dollars entre 1992 et $1995^{7}$.

Le bilan mitigé de ce genre d'opérations fort complexes a obligé le Conseil de sécurité de l'ONU à ajourner la préparation et la réalisation de grandes opérations, voire tout simplement à y renoncer (Burundi, République démocratique du Congo). Aujourd'hui, on donne la préférence à des missions plus restreintes et plus simples.

En 1997, le Conseil de sécurité a ainsi décidé de lancer une seule nouvelle mission: la Mission des Nations Unies au Guatemala (MINUGUA). Celle-ci allie

6. Roberts A., «The Crisis in UN Peacekeeping», in Crocker C.A. et al., Managing Global Chaos: Sources of and Responses to International Conflict, Washington D.C., 1996, pp. 297-319.

7. Ibid. 
de manière exemplaire un mandat humanitaire et un mandat de politique de sécurité sans estomper la limite entre ces deux domaines. A cette mission axée essentiellement sur les droits humains, on a adjoint pour trois mois 155 observateurs militaires. Ceux-ci avaient pour tâche exclusive de veiller au respect de l'accord d'Oslo du 4 décembre 1996 sur un cessez-le-feu définitif et de récolter les armes livrées par l'organisation rebelle URNG, pour les remettre au gouvernement. Une fois cette tâche accomplie, la mission civile s'est poursuivie seule ${ }^{8}$.

La mise en œuvre de la MINUGUA illustre la véritable mutation des interventions humanitaires au cours de la deuxième moitié des années 90: réduction de l'envergure des missions, limitation de la partie militaire et accent sur la coordination entre les diverses mesures. Alors que l'on évoquait beaucoup, au début des années 90, un «maintien de la paix robuste» accompagné de mesures civiles et que les mandats civils et militaires tendaient à se confondre (comme en Somalie), on considère à nouveau aujourd'hui que le rôle des militaires doit rester subordonné aux mesures diplomatiques et humanitaires, ne serait-ce que pour des raisons financières.

\section{- Croissance de l'aide humanitaire d'urgence et de l'aide à la reconstruction}

Il en va tout autrement pour l'aide humanitaire d'urgence et pour l'aide à la reconstruction. Dans ce domaine, les dépenses sont demeurées élevées et augmentent encore au niveau international. Cette croissance est surtout due à la crise dans le domaine du maintien de la paix. Dans nombre de cas, les opérations lancées ne sont en effet pas parvenues à imposer des cessez-le-feu précaires, à atténuer la violence dans les zones en conflit et à faire appliquer des accords de paix obtenus à l'arraché. Ces difficultés ont ouvert le débat sur l'adéquation des mandats aux crises, sur les limites des opérations de maintien de la paix et sur le rapport entre maintien de la paix et «imposition de la paix ${ }^{9}$. De plus, nombre de personnes ont réitéré la crainte de voir les activités humanitaires remplacer les actions issues d'une politique résolue ${ }^{10}$.

Le glissement du maintien de la paix au niveau international vers l'aide humanitaire a notamment eu pour conséquence d'engendrer des coûts internes dans les pays d'accueil ; des coûts qui dépassent largement les moyens à disposition pour 1'aide d'urgence sur place, voire ceux nécessaires à une opération de maintien de la paix. En effet, alors que les opérations de ce type sont financées par la communauté des Etats selon une clé de répartition précise, le coût de la prise en charge des réfugiés incombe à un nombre restreint de pays. Le passage à une «réhabilitation postconflit» se traduit ainsi par une certaine «désolidarisation» au niveau de la gestion internationale des conflits.

Le cas du Kosovo illustre on ne peut plus clairement les dilemmes des activités humanitaires. Alors que l'on n'avait pas du tout fait appel à la diplomatie préventive depuis le début des années 90 et que celle-ci s'est révélée peu efficace après l'éclatement de la crise, il paraissait peu judicieux d'attribuer un mandat

8. Findlay T., «Armed Conflict Prevention, Management and Resolution», in: Institut international de recherche sur la paix de Stockholm (éd.), SIPRI Yearbook 1998: Armaments, Disarmament and International Security, Oxford University Press, 1998, pp. 40 et suiv.

9. Kühne W. (éd.), Blauhelme in einer turbulenten Welt, Baden-Baden (Nomos), 1993.

10. Eisele M., «Im Auftrag des Sicherheitsrates: Friedensmissionen der Vereinten Nationen», in DGVN (éd.), Vereinte Nationen, $\mathrm{n}^{\circ}$ 1/février 1998, $46^{e}$ année, p. 6. 
classique de maintien de la paix (casques bleus) sur fond de combats entre l'Armée de libération du Kosovo (UCK) et les forces paramilitaires de Belgrade. Le Conseil de sécurité a fait ici preuve d'une retenue inhabituelle, d'une part en raison de l'opposition russe, mais surtout en raison de sa politique restrictive en matière de mandats. De leur côté, les Etats de l'OTAN n'étaient guère disposés à envoyer des troupes terrestres sur place. Sous la pression de l'ultimatum de l'OTAN, qui a menacé de lancer des attaques aériennes contre les positions militaires serbes, le négociateur américain Richard Holbrooke a pu conclure à la dernière minute un accord avec le président Milosevic sur le retrait des troupes serbes. Ces négociations n'ont toutefois pas permis de résoudre les principaux problèmes à l'origine de la crise, soit notamment la suppression de l'autonomie régionale par Belgrade.

L'aide humanitaire fournie ensuite avait des fondements pacifiques et éthiques. Tous les acteurs savaient toutefois pertinemment qu'à l'approche de l'hiver les villageois réfugiés dans les forêts allaient fuir à l'étranger et que l'aggravation de la crise humanitaire ne ferait que renforcer la position de l'UCK.

Certes, l'accord passé avec Belgrade a d'une part ouvert la porte à d'autres tractations diplomatiques. D'autre part, il a toutefois obligé la mission de l'OSCE et l'aide humanitaire d'urgence ainsi que l'aide à la reconstruction à opérer dans une zone non pacifiée ou pacifiée malgré elle. A l'heure actuelle (fin octobre 1998), il est dès lors difficile de prévoir l'évolution politique dans la région et une recrudescence des combats n'est pas à exclure. Il est tout aussi difficile d'évaluer l'efficacité de la menace de l'OTAN lorsque des activités humanitaires sont menées sur place.

\section{L'aide humanitaire}

dans les situations précaires et en tant qu'élément de l'économie de guerre

L'aide aux réfugiés dépend pour sa part directement de la sécurité qui règne dans les régions concernées. Les programmes se révèlent d'autant plus efficaces s'ils interviennent là où un processus de paix délibéré ou bien contrôlé permet de réduire l'insécurité existentielle. A l'opposé, des retards et des obstacles apparaissent inévitablement dans les régions où l'aide d'urgence doit être prolongée pour une durée illimitée en raison de la stagnation du processus de paix. C'est du moins ce que prouve la concentration des moyens humanitaires sur certaines zones en crise telles que la région des Grands Lacs en Afrique centrale, dans la Corne de l'Afrique et en Angola.

Dans certaines régions, le degré d'insécurité rend parfois toute opération d'aide humanitaire (pratiquement) impossible:

- Dans le nord du Caucase (Tchétchénie, Ingouchie, Ossétie du Nord), il n'a guère été possible d'acheminer une aide humanitaire à la population en raison de l'extrême précarité de la situation. La Suisse a ainsi dû se contenter de cofinancer les rares activités du CICR et du HCR qui restaient possibles.

- Dans nombre de régions en Afrique, le minimum de sécurité nécessaire à la réalisation de mandats humanitaires n'est pas garanti (la même remarque vaut aussi pour certaines régions de Colombie). Dans la région des Grands Lacs, les problèmes de sécurité ont gravement entravé les activités des organisations d'entraide malgré la gravité des carences nutritionnelles. Il faudrait 
en effet parvenir à démobiliser une large partie de l'armée pour pouvoir mettre en place une aide à la reconstruction qui pourrait déboucher sur une coopération à plus long terme.

La politique de sécurité comporte un autre phénomène - qu'il ne faut pas sousestimer d'ailleurs: il s'agit du risque de voir les prestations de l'aide internationale devenir partie intégrante de l'économie de guerre. Voici comment:

- épuisement de l'argent des opérations de grande envergure par la surévaluation délibérée de la monnaie du pays concerné;

- détournement de l'aide alimentaire par les parties en conflit et par les pays accueillant les flux de réfugiés au profit de l'armée et des milices;

- utilisation de l'aide alimentaire en tant qu'arme en la refusant aux groupes favorables à l'opposition;

- destruction des structures locales de pouvoir et de répartition par l'envoi massif d'aide (alimentaire) extérieure ${ }^{11}$.

Par ailleurs, les œuvres d'entraide - de taille moyenne avant tout - font état de corruption, de racket et d'autres intrigues et machinations de la part des «seigneurs de la guerre» ou, tout simplement, de bandes criminelles.

\section{$\square$ Tendance à privilégier la période après la crise}

Dans les pays où le processus de paix est en panne, tels l'Angola, dans les zones de guerre civile dont l'intensité va croissant (Afghanistan, Colombie, Grands Lacs, Sud du Soudan, Somalie) et dans les régions ayant surmonté la crise (Caucase du Nord), l'aide humanitaire ne pourra venir efficacement en aide aux populations dans le besoin que si des forces politiques et militaires sont chargées de protéger ses activités. Lorsque la sécurité n'est pas entièrement garantie - que ce soit en raison d'un échec de la diplomatie préventive, du manque d'efficacité d'une opération de maintien de la paix (voire de son absence) ou de la non application d'un accord de paix -, l'aide humanitaire pourra de moins en moins intervenir au milieu de la crise et devra attendre que le conflit s'apaise ${ }^{12}$.

Ce constat n'est pas nécessairement négatif. L'aide humanitaire peut en effet se révéler plus efficace et avoir des effets à plus long terme si elle intervient après la crise et non pas dans sa phase la plus aiguë. C'est notamment le cas lorsqu'il est possible d'assurer la transition entre l'aide humanitaire d'urgence et l'aide (également humanitaire) à la reconstruction et à la coopération à long terme dans le cadre de la coopération au développement. Ainsi, une intervention ciblée après la crise peut servir à prévenir d'autres crises et sauver ainsi plus de vies humaines qu'une aide d'urgence prodiguée dans des conditions précaires. Il ne faudrait toutefois pas en conclure qu'il faille suspendre toutes les prestations d'aide pendant les phases critiques d'une crise en raison des grands risques qui leur sont liés.

En fin de compte, le degré d'efficacité et la durabilité de l'aide dépendent pour beaucoup de la sécurité qui règne dans la région visée. Des situations graves peu-

11. Matthies V. (éd.), Vom Krieg zum Frieden. Kriegsbeendigung und Friedenskonsolidierung, Brême, 1995, pp. 68 et suiv.

12. Délégué à l'aide humanitaire et chef ASC, Jahresbericht 1997 der Abteilung Humanitäre Hilfe und SKH, Berne, mars 1998, p. 6. 
vent ainsi bloquer complètement la reconstruction ou la réintégration des réfugiés (Rwanda, putsch militaire en Sierra Leone). En Bosnie-Herzégovine, la reconstruction et l'aide humanitaire placées sous la responsabilité du Conseil de mise en œuvre de la paix, un organisme civil, ne sont possibles que sous la protection des troupes SFOR de l'OTAN, qui sont chargées de faire respecter l'accord de Dayton. Malgré l'envergure de cette mission militaire et civile, l'application de l'accord de paix ne progresse que lentement. Ainsi, nombre de personnes qui voudraient retourner chez elles (de leur propre gré) ne peuvent pas le faire car leur appartenance ethnique constitue une menace pour leur sécurité.

\section{$\square$ Les ajustements entre aide humanitaire et politique de sécurité}

On en conclura d'une part que l'aide humanitaire doit s'adapter au contexte défini par la politique de sécurité, mais d'autre part aussi que la situation humanitaire a modifié cette politique de sécurité.

La motivation humanitaire des interventions de l'ONU dans les régions en crise a mis fin aux orientations et aux influences qui caractérisaient naguère les opérations de maintien de la paix. En effet, des arguments humanitaires ne permettent guère de justifier des préférences régionales. De plus, la légalisation croissante de la politique de sécurité exige que les crises humanitaires reçoivent des traitements équivalents. Cette évolution, qui plonge ses racines dans les crises rwandaise et bosniaque, s'est notamment traduite par la création du Tribunal pénal international (TPI) à Rome. Même si la compétence juridictionnelle de cette cour ne répond pas encore à toutes les exigences d'une époque marquée par des conflits internes et le démembrement d'Etats, ses pouvoirs s'étendent néanmoins à quatre grands délits: génocide, crime contre l'humanité, crime de guerre et guerre d'agression ${ }^{13}$. L'histoire nous dira si les mécanismes prévus pour mettre en marche cette machine pénale réduiront le nombre de crises humanitaires provoquées par les conflits armés.

Si le Conseil de sécurité s'en tient à sa nouvelle orientation, il est peu probable que l'on assiste à la mise sur pied d'opérations de l'envergure de la FORPRONU dans l'ex-Yougoslavie. La crise actuelle du maintien de la paix risque plutôt de déboucher sur une multiplication des activités civiles et humanitaires... avec tous les risques que cela comporte.

La Mission de vérification au Kosovo (KVM) de l'OSCE est l'exemple le plus récent de ce changement. Cette mission, qui réunit 2000 civils et militaires non armés, se fonde en effet sur de nouveaux critères nés de l'urgence. La KVM a ainsi pour tâche de vérifier l'observation du cessez-le-feu, le retrait des forces de sécurité yougoslaves, le retour des personnes déplacées et des réfugiés, ainsi que l'engagement de négociations entre Belgrade et Pristina. Elle soutiendra par ailleurs des organisations humanitaires et veillera à la tenue d'élections au Kosovo $^{14}$. Dans ce sens, la KVM se veut une opération civile pour favoriser la résolution du conflit. Mais cette opération dépend de la pression militaire entretenue par l'OTAN.

13. Kaul H.P., «Der Internationale Strafgerichtshof: Das Ringen um seine Zuständigkeit und Reichweite», in Humanitäres Völkerrecht, $\mathrm{n}^{\circ} 3,1998$, Themenheft Internationaler Strafgerichtshof, DRK, pp. 138 et suiv.

14. Département fédéral des affaires étrangères, «Participation suisse à la Mission de vérification de l'OSCE au Kosovo», Communiqué de presse, Berne, 21 octobre 1998. 
Aucune mission civile humanitaire ne pourra en effet assurer la protection des habitants du Kosovo tant que la situation dans la région ne se sera pas stabilisée. Or, cette stabilisation dépend à son tour d'un vaste processus de paix qui ne peut pas se limiter au retour des réfugiés et des personnes en fuite.

\section{UN CORPS DE SOLIDARITÉ POUR APPUYER LA POLITIQUE DE SÉCURITÉ HUMANITAIRE DE LA SUISSE?}

Avant la crise de 1'ex-Yougoslavie, la Suisse n'a jamais consacré autant de moyens à une région en conflit. Entre 1991 et 1996, ses dépenses (l'aide aux réfugiés en Suisse comprise) ont en effet atteint 1,2 milliard de dollars ${ }^{15}$.

L'aide suisse destinée à la Bosnie-Herzégovine comprenait les domaines suivants: aide financière, aide humanitaire, coopération technique, campagne pour le retour des réfugiés, soutien à l'organisation d'élections, bérets jaunes, missions de l'OSCE, soutien à des médias indépendants, etc. Cette diversité reflète la tendance internationale dans la mesure où la Suisse ne possède pas son propre contingent de maintien de la paix et que son aide humanitaire passe par un ensemble d'autres mesures telles que l'envoi d'observateurs militaires non armés, le soutien à des policiers civils (CIVPOL), les interventions du Corps suisse d'aide en cas de catastrophe (ASC) et de l'Office fédéral des réfugiés (ODR). Depuis la conclusion du Traité d'Ottawa, en décembre 1997, la Suisse accorde une place croissante au déminage et à l'aide aux victimes des mines antipersonnel. La campagne de déminage est financée notamment par la DDC, par l'Office fédéral des réfugiés et par le DDPS.

Dans ce contexte, le rapport de la Commission d'étude pour les questions stratégiques («Rapport Brunner») met en toute logique l'accent sur l'intégration de la Suisse dans le système de sécurité international. Le rapport souligne que la Suisse - comme ses voisins d'ailleurs - n'est plus guère menacée d'une agression militaire du type classique. Pour ce qui est de se protéger contre les risques indirects inhérents à des crises plus ou moins proches du point de vue géographique - comme celles de Bosnie ou du Kosovo -, la Suisse ne peut pas continuer sur la voie solitaire. Elle devra collaborer avec ses voisins à la réalisation d'un espace de sécurité élargi et indivisible. C'est pourquoi la commission recommande entre autres la création d'un Corps suisse de solidarité «doté de professionnels et de miliciens (volontaires) spécialement entraînés et équipés, et capable d'intervenir dans des missions de secours, de paix et d'appui aux civils $\gg\left(\right.$ recommandation $\mathrm{n}^{\circ}$ 9).

Les prises de position ayant suivi la publication du rapport Brunner, de même que divers sondages d'opinion, montrent que la majorité de la population est favorable à une politique de sécurité humanitaire, voire à ce que la Suisse joue un rôle plus actif dans les opérations de maintien de la paix. Certains remettent néanmoins en question la création du Corps suisse de solidarité au sein du DDPS. Est-ce vraiment là le moyen le plus approprié pour améliorer l'image de la Suisse dans le domaine de la promotion de la paix au niveau international ? $^{16}$

15. L'aide humanitaire de la Suisse est relativement élevée en comparaison internationale puisqu'elle représente 19 à $20 \%$ de l'aide publique totale au développement. Elle est néanmoins restée stable ces dix dernières années. Voir Annuaire Suisse-Tiers Monde 1998, n 17, IUED, Genève, 1998, pp. 321 et suiv.

16. DDPS, Groupe de coordination Armée 200X (éd.), Résultats de la consultation relative au rapport Brunner, Berne, 18.8.1998. 
Le principal reproche formulé à l'encontre du Corps suisse de solidarité est que celui-ci ne servirait pas à prévenir de futures menaces, mais à y réagir sur les plans technique et militaire. Le Corps de solidarité a ainsi inévitablement été associé à la fourniture de couvertures en laine, aux soins sanitaires, au transport, à la logistique et à l'aide en cas de catastrophe, mais jamais à la diplomatie préventive, aux communications, aux bons offices et à la promotion multilatérale de la paix - des domaines qu'il convient de privilégier d'urgence ${ }^{17}$. D'aucuns craignaient également que le Corps de solidarité du DDPS ne vienne concurrencer directement l'ASC ou ne veuille même l'absorber. Enfin, il faut bien admettre que les questions concernant le coût, l'efficacité ainsi que la place de cette nouvelle entité dans les multiples activités déjà existantes (CIVPOL, observateurs militaires, etc.) n'ont pas reçu de réponses satisfaisantes.

En bref, un Corps de solidarité n' aura guère d'effets s'il ne s'inscrit pas dans un cadre politique plus large qui vise à promouvoir la paix et qui comprend des activités de prévention et de gestion des crises. Si la Suisse souhaite vraiment se profiler parmi les acteurs, désormais puissants et compétents, qui œuvrent pour la promotion de la paix, elle doit se doter d'une stratégie cohérente et ne pas se contenter d' "opérations » isolées. Dans ce domaine, comme au niveau national, c'est toujours la politique qui prime. La participation de la Suisse aux efforts internationaux de promotion de la paix passe inévitablement par une diplomatie préventive et de médiation, qui s'inscrive dans le contexte international. Il appartient donc à la politique extérieure d'identifier les foyers de crise où une intervention de la Suisse a des chances de réussir. Elle doit ensuite définir les instruments nécessaires à cette intervention et coordonner leur mise en œuvre. Dans ce cadre, le Corps de solidarité pourrait jouer un rôle important, au même titre que la coopération préventive au développement, que l'aide humanitaire et que les prestations d'aide - en hausse constante - des organisations non gouvernementales. La création d'un tel corps ne se justifie que dans le cadre d'une stratégie de gestion des conflits constructive, prévue sur le moyen et le long terme; une stratégie fondée non seulement sur la politique extérieure et sur la politique de développement, mais aussi sur les orientations d'autres pays partenaires.

\section{DE L'AIDE D'URGENCE À LA PRÉVENTION}

$\mathrm{Au}$ niveau international, tous les intervenants s'accordent sur un point: la prévention ne pourra être efficace que si les moyens limités alloués à la politique de paix ne sont pas tous consacrés à l'aide d'urgence et aux interventions en cas de crise.

A l'occasion d'une conférence des Nations Unies intitulée «Preventive Diplomacy: The Therapeutics of Mediation", qui s'est tenue à New York du 23 au 24 avril 1996, Jan Eliasson, secrétaire d'Etat au Ministère suédois des affaires étrangères, a déclaré que pour demeurer une organisation mondiale efficace l'ONU se devait d'élaborer des instruments prometteurs de politique préventive. Il a parlé d'un «impératif de la diplomatie préventive» qui aurait pour objectif

17. Prise de position des collaborateurs et collaboratrices de l'Institut pour la résolution de conflits de la Fondation suisse pour la Paix. 
d' "économiser des milliards de dollars et de sauver des vies humaines ainsi que l'ONU elle-même». Il estime en effet que l'Organisation des Nations Unies traverse une grave crise qui remet en cause à la fois son financement, son identité et ses valeurs. L'organisation ne peut donc pas se permettre de lancer un nombre élevé d'opérations de maintien de la paix de grande envergure.

La diplomatie préventive, beaucoup moins coûteuse, acquiert ainsi une importance d'autant plus cruciale parmi les instruments de maintien de la paix à la disposition des Nations Unies. Tout le monde sait que prévenir vaut mieux que guérir. De même, une politique préventive est plus humaine, plus sensée et bien moins coûteuse qu'une intervention a posteriori qui comprend l'apaisement du conflit et le stationnement de troupes de maintien de la paix. Si le Conseil de sécurité prend au sérieux le rôle du Tribunal pénal international mentionné cidessus, il pourra contribuer grandement à la prévention des crises humanitaires. En effet, sur la base des mécanismes mis en place et en vertu du chapitre VII de la Charte des Nations Unies, il pourra renvoyer à ce tribunal des situations faisant craindre de graves infractions. Mais ce n'est là que l'aspect juridique de la prévention. Au-delà, il s'agit de mettre au point un véritable programme politique comprenant la prévention opérationnelle (à court terme) et structurelle (à long terme $)^{18}$.

Toutefois, même si ce programme voit le jour et est appliqué, la prévention des crises n'obtiendra pas toujours des résultats probants dans les multiples crises et conflits, du moins pas dans un avenir prévisible. Or, en cas d'échec de la prévention, l'aide humanitaire ne doit pas servir automatiquement de solution d'urgence. Il convient au contraire de sortir le maintien de la paix de sa crise et de tenir compte, lors des prochaines interventions humanitaires, des leçons tirées en Bosnie, en Somalie, au Kosovo et dans d'autres zones en conflit. Entre les deux extrêmes que furent la FORPRONU et la MINUGUA, le Conseil de sécurité dispose en effet de tout un éventail de moyens d'action.

L'expérience montre que la gestion des crises et des conflits doit être parfaitement crédible du point de vue politique. Voici quelques principes qui ressortent de diverses discussions et qui indiquent la voie à suivre:

- Une politique qui vise résolument à éviter ou à atténuer la souffrance doit - tout comme l'aide d'urgence - s'appliquer en fonction du principe d'égalité de traitement et non pas selon des préférences régionales.

- Pour pouvoir réprimer des injustices humanitaires, l'organisation ou l'Etat tiers doit fonder son intervention sur l'impartialité et sur la morale.

- Les moyens mis en œuvre pour atteindre l'objectif visé doivent respecter le principe de l'adéquation et de la proportionnalité. Il faut coordonner mais non pas confondre le maintien de la paix et l'aide d'urgence.

- Une intervention militaire basée sur des motifs humanitaires ne doit être envisagée que lorsque tous les moyens de régler le différend par la voie pacifique auront été épuisés.

18. Voir entre autres Carnegie Commission on Preventing Deadly Conflict (éd.), Preventing Deadly Conflict: Final Report with Executive Summary, New York, décembre 1997; Report of The Aspen Institute Conference on Conflict Prevention: Strategies to Sustain Peace in the Post-Cold War World, du 30 juillet au 3 août 1996, Aspen, Colorado. 
- Une telle intervention doit constituer un moindre mal, c'est-à-dire que les avantages escomptés pour les parties concernées et pour la communauté internationale doivent dépasser les inconvénients prévisibles.

- L'objectif d'une intervention humanitaire est de réussir et de protéger les victimes. Elle doit être limitée, méthodique et avoir donc presque le caractère d'une intervention policière.

- On doit disposer de la certitude morale que l'intervention permettra d'atténuer ou d'éviter de graves souffrances humaines.

La Suisse dispose de diverses instances pour remplir ces critères: DDC, ASC, aide aux réfugiés, promotion de la paix, experts en matière de droits humains, etc. Un nouveau Corps de solidarité devrait donc surtout venir combler les lacunes qui subsistent dans le domaine du maintien de la paix lors d'une intervention humanitaire.

Pour sa part, la future Fondation Suisse solidaire pourrait constituer le moyen adéquat de promouvoir une culture politique de la prévention. Grâce à ses activités visant à prévenir la pauvreté et la marginalisation, à éviter les conflits armés et à réconcilier les parties en conflit, elle pourrait contribuer efficacement à combler les lacunes de la politique préventive ${ }^{19}$.

\section{Sigles}

$\begin{array}{ll}\text { ASC } & \text { Corps suisse d'aide en cas de catastrophe } \\ \text { BMZ } & \begin{array}{l}\text { Bundesministerium für Zusammenarbeit - } \\ \text { Ministère pour la coopération économique (Rép. féd. d'Allemagne) }\end{array} \\ \text { CIVPOL } & \begin{array}{l}\text { Police civile } \\ \text { FAST }\end{array} \\ \begin{array}{ll}\text { Frühanalyse von Spannungen und Tatsachenermittlung } \\ \text { (Projet de l'Institut pour la résolution de conflits, Fondation suisse pour la Paix) }\end{array} \\ \text { FORPRONU } & \text { Force de protection des Nations Unies } \\ \text { HCR } & \text { Haut-Commissariat des Nations Unies pour les réfugiés } \\ \text { KVM } & \text { Mission de vérification au Kosovo } \\ \text { MINUGUA } & \text { Mission des Nations Unies au Guatemala } \\ \text { OCDE } & \text { Organisation de coopération et de développement économiques } \\ \text { ODR } & \text { Office fédéral des réfugiés } \\ \text { OSCE } & \text { Organisation pour la sécurité et la coopération en Europe } \\ \text { OTAN } & \text { Organisation du Traité de l'Atlantique Nord } \\ \text { SFOR } & \text { Stabilization force } \\ \text { TPI } & \text { Tribunal pénal international } \\ \text { UCK } & \text { Armée de libération du Kosovo } \\ \text { URNG } & \text { Unidad Revolucionaria Nacional Guatemalteca } \\ \text { ZPNU } & \text { Zones protégées par les Nations Unies }\end{array}$

19. Département fédéral des finances, Loi sur la «Fondation Suisse solidaire». Projet pour la procédure de consultation, Berne, juin 1998. 